\title{
Validation of GOCE gravity field models by means of orbit residuals and geoid comparisons
}

\author{
Th. Gruber • P. N. A. M. Visser • Ch. Ackermann • \\ M. Hosse
}

Received: 8 October 2010 / Accepted: 17 May 2011 / Published online: 8 June 2011

(C) Springer-Verlag 2011

\begin{abstract}
Three GOCE-based gravity field solutions have been computed by ESA's high-level processing facility and were released to the user community. All models are accompanied by variance-covariance information resulting either from the least squares procedure or a Monte-Carlo approach. In order to obtain independent external quality parameters and to assess the current performance of these models, a set of independent tests based on satellite orbit determination and geoid comparisons is applied. Both test methods can be regarded as complementary because they either investigate the performance in the long wavelength spectral domain (orbit determination) or in the spatial domain (geoid comparisons). The test procedure was applied to the three GOCE gravity field solutions and to a number of selected pre-launch models for comparison. Orbit determination results suggest, that a pure GOCE gravity field model does not outperform the multi-year GRACE gravity field solutions. This was expected as GOCE is designed to improve the determination of the medium to high frequencies of the Earth gravity field (in the range of degree and order 50 to 200). Nevertheless, in case
\end{abstract}

Th. Gruber $(\bowtie) \cdot$ Ch. Ackermann $\cdot$ M. Hosse

Institute of Astronomical and Physical Geodesy,

Technische Universität München, Arcisstrasse 21,

80333 Munich, Germany

e-mail: Thomas.Gruber@tum.de

Ch. Ackermann

e-mail: Christian.Ackermann@tum.de

M. Hosse

e-mail: Michael.Hosse@tum.de

P. N. A. M. Visser

Delft Institute of Earth Observation and Space Systems (DEOS),

Delft University of Technology, Kluyverweg 1, 2629 HS Delft,

The Netherlands

e-mail: p.n.a.m.visser@tudelft.nl of an optimal combination of GOCE and GRACE data, orbit determination results should not deteriorate. So this validation procedure can also be used for testing the optimality of the approach adopted for producing combined GOCE and GRACE models. Results from geoid comparisons indicate that with the 2 months of GOCE data a significant improvement in the determination of the spherical harmonic spectrum of the global gravity field between degree 50 and 200 can be reached. Even though the ultimate mission goal has not yet been reached, especially due to the limited time span of used GOCE data (only 2 months), it was found that existing satellite-only gravity field models, which are based on 7 years of GRACE data, can already be enhanced in terms of spatial resolution. It is expected that with the accumulation of more GOCE data the gravity field model resolution and quality can be further enhanced, and the GOCE mission goal of 1-2 cm geoid accuracy with $100 \mathrm{~km}$ spatial resolution can be achieved.

Keywords GOCE · Gravity Field · Validation - Orbit . Geoid

\section{Introduction}

Based on the first full repeat cycle of GOCE data (November 2009 to early January 2010) three independent gravity field solutions have been computed by the GOCE science processing system (High level Processing Facility-HPF, Rummel et al. 2004). These models were made available to the GOCE user community in July 2010. The three models differ by the processing strategies applied and the level of a-priori knowledge introduced. Details of the processing characteristics are summarized in Pail et al. (2011). 
Basically, the major characteristics are that one solution starts with a GRACE/altimetric/terrestrial combination model and uses GOCE data to further enhance the global gravity field (GOCE-DIR), another technique applies to some extent long wavelength information from a GRACE-only gravity field and combines this with GOCE data (GOCE-SPW), while the third model fully relies on GOCE data and does not use a-priori information at all (GOCE-TIM). All models deliver quality information in terms of variance-covariance matrices resulting from a least squares solution or a Monte-Carlo approach. This gives a good impression about the performance and error characteristics of the models, but will not give a final answer about the precision one can reach with the 2 months of GOCE data. For this reason, an external validation needs to be done in order to give a deeper insight into the precision of these models. For this purpose, the GOCE (and other) models (see Table 1) are compared to external independent information or are further used to derive other geophysical quantities. An example of the latter is the determination of the dynamic ocean topography (DOT) from the GOCE models. It is referred to Knudsen et al. (2011) for some DOT results based on GOCE geoids. In this work, we try to determine the precision of the GOCE models by means of orbit determination for a selected set of low and high Earth orbiting satellites (Sect. 2) and geoid comparisons (Sect. 3). While orbit determination provides a closer look into the spectral domain of a gravity field, geoid comparisons investigate the spatial error characteristics. It shall be noted already at this point, that each of the validation procedures might provide different results. Therefore, in order to draw the correct conclusions from these tests, they have to be regarded in a common view. A summary of the results and an attempt to classify the obtained validation results is provided in Sect. 4.

\section{GOCE gravity field validation by means of orbit determination}

High-accuracy gravity field models are a prerequisite for precise orbit determination (POD) of low Earth orbiting (LEO) satellites. Vice-versa, POD of LEO satellites can be used for validating the quality of produced gravity field models. Due to the relatively quick dampening of orbit perturbations with increasing spatial resolution, the validation of such models by POD is limited in terms of spatial resolution or it might be stated that POD is especially suited to test the quality at the longer wavelengths. Nevertheless, because of the very high precision of orbit determination achievable today-of the order of a few centimetres with modern tracking systems such as the Global Positioning System (GPS) - higher and higher spatial resolution scales of the Earth's gravity field can be observed or distinguished by POD methods. Therefore, testing of GOCE gravity field solutions by POD of LEO satellites is an integral part of the overall validation scheme. This testing has been done for a selection of satellites with altitudes ranging from about 450 to about $6,000 \mathrm{~km}$. These satellites are ERS-2, CHAMP, GRACE-A/B and LAGEOS$1 / 2$ for which a mature POD system has been implemented at DEOS allowing orbit determination with precisions at the few cm level (Scharroo and Visser 1998; Visser et al. 2002; van den IJssel and Visser 2003; Van Helleputte and Visser 2007; Noomen et al. 1998). The orbit computations are done with the NASA Goddard Space Flight Center (GSFC) GEODYN software (Pavlis et al. 2006). In fact, also POD tests were conducted for GOCE itself. It is fair to assume that certain resonant perturbations for GOCE are not well represented by pre-launch models. It is interesting to assess if such resonances are picked up better by the first release of GOCE gravity field models.

Table 1 Global gravity field models used in this study

\begin{tabular}{lcll}
\hline Name & Maximum D/O & Description & References \\
\hline GOCE-DIR $^{\mathrm{a}}$ & 240 & GOCE direct approach (EIGEN-5C and GOCE) & ESA 2010 \\
& & & Bruinsma et al. 2010 \\
GOCE-TIM & GOCE time-wise approach (GOCE-only) & ESA 2010 & Pail et al. 2010 \\
& 224 & & ESA 2010 \\
GOCE-SPW & & GOCE space-wise approach (GOCE-only plus GRACE for low degrees) & Migliaccio et al. 2010 \\
& 210 & & Mayer-Gürr et al. 2010 \\
ITG-GRACE2010S & 180 & 7 years GRACE data & Förste et al. 2008 \\
EIGEN-5S & 150 & 5 years GRACE data & Förste et al. 2008 \\
EIGEN-5C & 360 & EIGEN-5S, surface gravimetry, altimetry & Pavlis et al. 2008
\end{tabular}

Maximum D/O specifies the maximum degree and order of these models

a Official GOCE product name: EGM_GOC_2_20091101T000000_20100110T235959_0002

${ }^{b}$ Official GOCE product name: EGM_GOC_2_20091101T000000_20100111T000000_0002

c Official GOCE product name: EGM_GOC_2_20091030T005757_20100111T073815_0002 
The precise orbit determinations are based on different combinations of tracking data and on representative time periods (Table 2). For all POD implementations, use is made of state-of-the-art standards, reference frame and station coordinate solutions (IERS 2008; Altamimi et al. 2007) and dynamic force modelling. More details about the POD setup and results for several satellites are included below. In all cases, first orbit computations were done with the satellite-only EIGEN-5S gravity field model (Förste et al. 2008), which served as the reference pre-launch model. Second, all the orbits were recomputed with the first series of released GOCE models derived by the direct, time-wise and space-wise methods, in the following referred to by the identifiers GOCE-DIR, GOCE-TIM and GOCE-SPW, respectively. Third, gravity field models were truncated at degree

Table 2 Tracking data types and periods for the selected satellites

\begin{tabular}{|c|c|}
\hline ERS-2 & $\begin{array}{l}\text { SLR, PRARE, altimeter crossover observations } \\
\text { 7-day arcs } \\
2 \text { January } 1996-6 \text { January } 1997(\approx 1 \text { year }) \\
l_{\max }=120\end{array}$ \\
\hline CHAMP & $\begin{array}{l}\text { GPS SST } \\
\text { Accelerometer observations } \\
\text { Daily orbital arcs } \\
20-30 \text { May } 2001 \\
l_{\max }=200\end{array}$ \\
\hline GRACE-A/B & $\begin{array}{l}\text { GPS SST } \\
\text { Accelerometer observations } \\
\text { Daily orbital arcs } \\
1-10 \text { August } 2002 \\
l_{\max }=200\end{array}$ \\
\hline LAGEOS-1/2 & $\begin{array}{l}\text { SLR observations } \\
\text { 8-day arcs (1-day overlaps between consecutive arcs) } \\
5 \text { January-29 December } 2002(\approx 1 \text { year }) \\
l_{\max }=20\end{array}$ \\
\hline GOCE & $\begin{array}{l}\text { GPS SST } \\
\text { Common-mode accelerometer observations } \\
\text { Daily orbital arcs } \\
20-29 \text { April } 2010 \\
l_{\max }=200\end{array}$ \\
\hline
\end{tabular}

The orbital arc lengths and the maximum spherical harmonic degree $\left(l_{\max }\right)$ used in the orbit determinations are indicated as well and order 200 (the full model was used if the associated maximum degree is lower than 200) for the POD of the CHAMP, GRACE and GOCE satellites. For the POD of the ERS-2 satellite, which flies at a significantly higher altitude, a maximum degree of 120 was applied. Finally, for the POD of the LAGEOS satellites, with an orbital altitude of about $5,900 \mathrm{~km}$, the gravity field models were truncated at degree and order 20.

For ERS-2 with an altitude of about $800 \mathrm{~km}$, standard POD setups are used involving the estimation of many atmospheric drag scaling coefficients and empirical accelerations to absorb especially non-gravitational modelling errors (Scharroo and Visser 1998). In principle, this would make the ERS-2 POD less sensitive to gravity field modelling errors and less suitable for validation since such errors can be partly absorbed by these estimated parameters. However, the ERS-2 POD is still sensitive to gravity field model errors, as was e.g. shown by Visser (2005). This is also reflected by the results included in Table 3. The Root-Mean-Square (RMS) of fit of ERS-2 Satellite Laser Ranging (SLR), Precise-Range and Range-rate Equipment (PRARE) range (RNG) and rangerate (RR), single-satellite (SXO) and ERS-2/TOPEX dualsatellite altimeter crossover (DXO) observations slightly deteriorates when using the GOCE combined GOCE-DIR and GOCE-SPW models and significantly deteriorates when using the (GOCE-only) GOCE-TIM model (e.g. the SLR fit increases from 4.3 to $5.0 \mathrm{~cm}$ ). This is an indication that the long-wavelengths of the GOCE gravity field models are not an improvement over the pre-launch EIGEN-5S model. This is further supported by a more detailed analysis of the altimeter crossover residuals, which not only reflect sea level variations and possibly media correction errors (such as tropospheric and ionospheric delay), but also radial orbit error. These residuals are averaged in geographical $2^{\circ} \times 2^{\circ}$ bins and reflect the mean ERS-2 minus TOPEX (DXO) and mean ERS-2 ascending minus descending (SXO) geographically correlated radial orbit errors (Scharroo and Visser 1998). Although these errors are for all used gravity field models at a low level of just a few $\mathrm{cm}$, an increase can be observed for the GOCE models, especially again for the GOCE-TIM model, which was produced from scratch using only 2 months of GOCE data (Table 4).

The geographical patterns of the correlated radial orbit errors are displayed in Figs. 1 and 2 for the ERS-2
Table 3 RMS-of-fit of tracking observations for ERS-2 with different gravity field models

\begin{tabular}{llllll}
\hline Model & SLR $(\mathrm{cm})$ & PRARE RNG $(\mathrm{cm})$ & PRARE RR $(\mathrm{mm} / \mathrm{s})$ & SXO $(\mathrm{cm})$ & DXO $(\mathrm{cm})$ \\
\hline EIGEN-5S & 4.3 & 4.1 & 0.25 & 6.7 & 6.9 \\
GOCE-DIR & 4.3 & 4.2 & 0.25 & 6.8 & 6.9 \\
GOCE-TIM & 5.0 & 4.6 & 0.28 & 7.3 & 7.4 \\
GOCE-SPW & 4.4 & 4.2 & 0.25 & 6.8 & 6.9 \\
\hline
\end{tabular}


Table 4 RMS (cm) of geographically averaged ERS-2 single- (SXO) and ERS-2/TOPEX dual-satellite altimeter crossover (DXO) residuals with different gravity field models

\begin{tabular}{lllll}
\hline & EIGEN-5S & GOCE-DIR & GOCE-TIM & GOCE-SPW \\
\hline${\text { SXO } 2^{\circ} \times 2^{\circ}}^{\circ} 2.1$ & 2.5 & 3.3 & 2.2 \\
${\text { DXO } 2^{\circ} \times 2^{\circ}}^{1} 1.9$ & 1.9 & 2.9 & 1.9 \\
\hline
\end{tabular}

single-satellite and ERS-2/TOPEX dual-satellite altimeter crossover observations, respectively, both for the pre-launch EIGEN-5S and GOCE-only GOCE-TIM model. The larger ERS-2 radial orbit error with the latter model is manifested as more pronounced track patterns.

A more rigorous testing of the GOCE gravity field models by POD can be done by using very precise reduced-dynamic CHAMP and GRACE-A/B orbit solutions, which have a claimed precision level of a few $\mathrm{cm}$ (e.g. Van Helleputte and Visser 2007). These orbit solutions are converted to Cartesian $X, Y$ and $Z$ coordinates in the inertial Earthcentered reference frame, which then serve as observations in a fully dynamic orbit determination. CHAMP and GRACE are orbiting at relatively low altitudes $(400-550 \mathrm{~km})$ and for these altitudes, non-gravitational force models are not very precise compared to the quality of recent gravity field models. Just like with ERS-2, the estimation of drag scaling coefficients and empirical accelerations would be required to absorb associated modeling errors. However, the nongravitational force modeling can be completely replaced by the available high-precision accelerometer observations. For CHAMP, these observations are less precise than for the GRACE satellites, which carry newer generation accelerometers. Moreover, CHAMP provides only precise accelerometer observations for two directions due to the failure of an electrode for the Z-predominantly radial-direction. When using accelerometer observations instead of nongravitational force models, a number of additional parameters needs to be estimated, namely accelerometer bias and scale factors for each (used) axis. The $X$ and $Y$ axes of all accelerometers are predominantly aligned with the flight and cross-track directions.
Fig. 1 Mean of ERS-2 singe-satellite altimeter crossover residuals averaged in $2^{\circ} \times 2^{\circ}$ bins for the EIGEN-5S (top) and GOCE-TIM (bottom) gravity field models
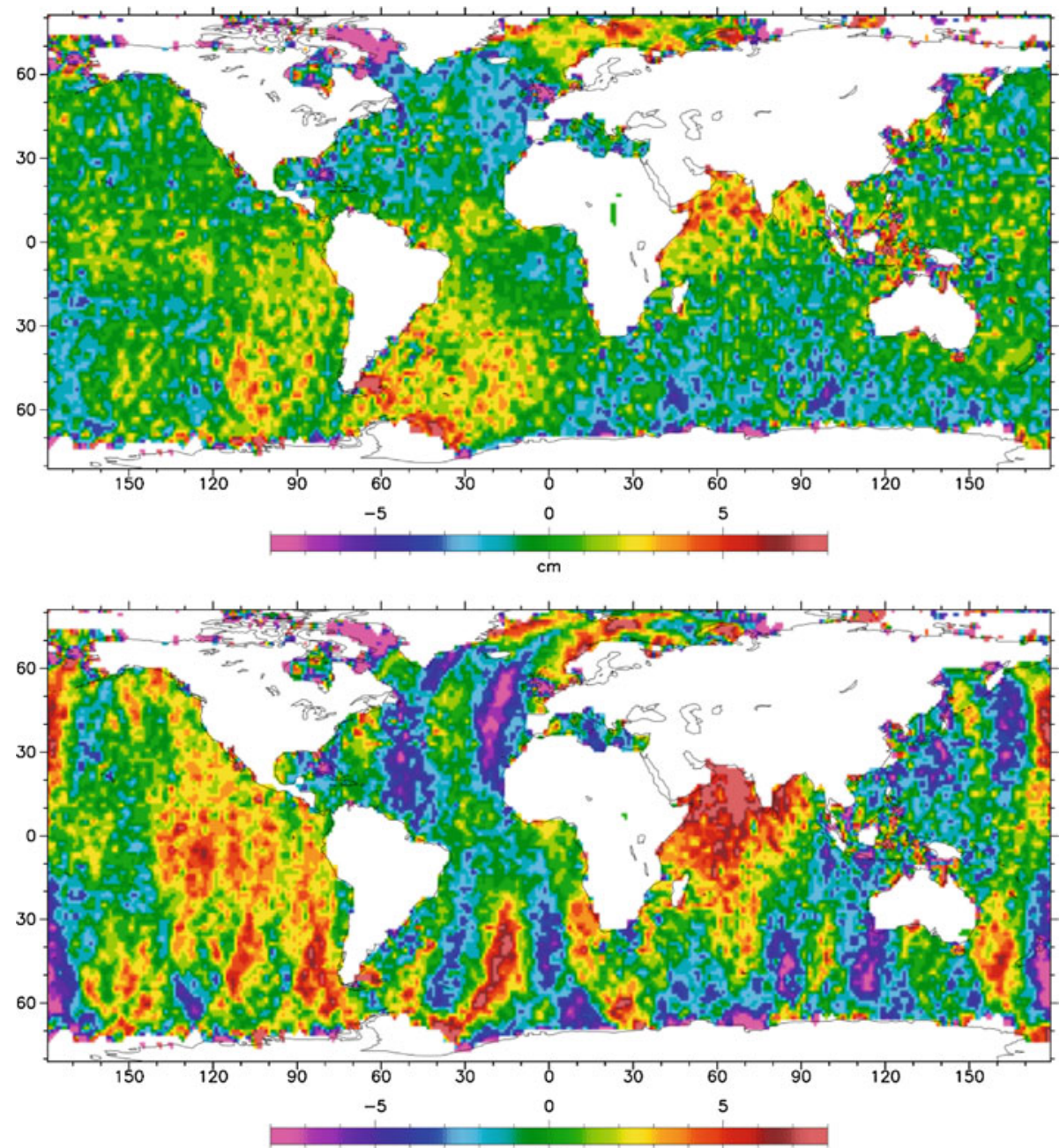

$\mathrm{cm}$ 
Fig. 2 Mean of ERS-2/TOPEX dual-satellite altimeter crossover residuals averaged in $2^{\circ} \times 2^{\circ}$ bins for the EIGEN-5S (top) and GOCE-TIM (bottom) gravity field models
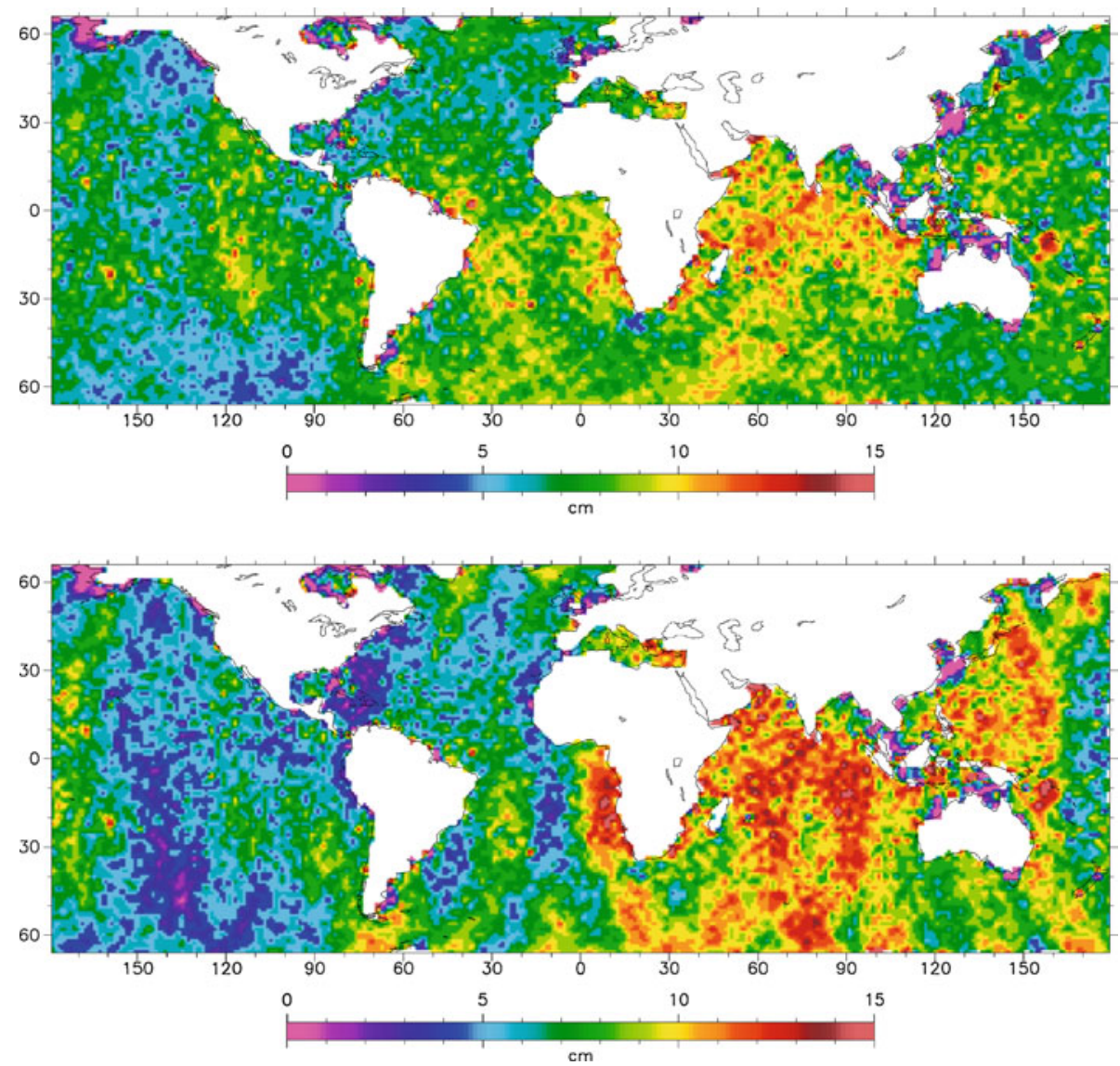

For CHAMP and GRACE, the POD tests are done for 1-day orbital arcs. Fully dynamic POD for CHAMP is defined as the estimation of only 13 parameters: initial position (3) and velocity (3) at start time, 2 bias and scale factors for the accelerometer X and Y axes (4), and 3 empirical accelerations (one radial constant and one set of 1 cycle-per-orbitalrevolution (cpr) radial sine/cosine coefficients) to account for the missing radial direction. For GRACE A and B, the same parameter set is estimated, except the 3 empirical accelerations are replaced by the additional bias and scale factor for the accelerometer $Z$ axis (totaling 12 estimated parameters). The RMS-of-fit by this POD approach and setup of the coordinates from the reduced-dynamic orbit solutions is displayed in Table 5. For GRACE-A/B, a 3-dimensional fit of the order of $8 \mathrm{~cm}$ is achieved with the pre-launch EIGEN$5 \mathrm{~S}$ model compared to about $40 \mathrm{~cm}$ for CHAMP (reflecting the lower quality of the accelerometer and missing 3rd direction). The GOCE-DIR and GOCE-SPW gravity field models lead to a significant increase of this RMS-of-fit and the GOCE-TIM model to an even further deterioration. These results reflect the lower performance of GOCE at the long wavelengths.

As a consistency test, also fully dynamic orbit determinations were conducted for GOCE, where the reduced-dynamic Rapid Science Orbit (RSO) position coordinates were used
Table 5 RMS-of-fit of reduced-dynamic orbit solutions for prior and GOCE gravity field models

\begin{tabular}{llrrrr}
\hline Satellite & Model & $X(\mathrm{~cm})$ & $Y(\mathrm{~cm})$ & $Z(\mathrm{~cm})$ & $3 \mathrm{D}(\mathrm{cm})$ \\
\hline CHAMP & EIGEN-5S & 24.2 & 17.9 & 28.5 & 41.4 \\
GRACE-A & EIGEN-5S & 4.9 & 3.8 & 4.8 & 7.8 \\
GRACE-B & EIGEN-5S & 4.8 & 3.7 & 4.6 & 7.6 \\
GOCE & EIGEN-5S & 10.6 & 11.8 & 15.7 & 22.3 \\
CHAMP & GOCE-DIR & 25.5 & 19.1 & 30.0 & 43.8 \\
GRACE-A & GOCE-DIR & 6.7 & 5.3 & 6.4 & 10.7 \\
GRACE-B & GOCE-DIR & 6.2 & 5.1 & 6.0 & 10.0 \\
GOCE & GOCE-DIR & 8.7 & 9.6 & 11.7 & 17.4 \\
CHAMP & GOCE-TIM & 109.6 & 160.3 & 33.6 & 197.1 \\
GRACE-A & GOCE-TIM & 78.4 & 86.0 & 48.2 & 126.0 \\
GRACE-B & GOCE-TIM & 77.9 & 85.3 & 48.6 & 125.3 \\
GOCE & GOCE-TIM & 13.7 & 15.7 & 18.7 & 28.0 \\
CHAMP & GOCE-SPW & 24.7 & 18.3 & 27.6 & 41.3 \\
GRACE-A & GOCE-SPW & 10.3 & 6.1 & 7.1 & 13.9 \\
GRACE-B & GOCE-SPW & 10.7 & 6.6 & 7.5 & 14.6 \\
GOCE & GOCE-SPW & 11.2 & 12.2 & 15.1 & 22.4 \\
\hline
\end{tabular}

as observations which have a precision of the order of $4 \mathrm{~cm}$ (Visser et al. 2009; Bock et al. 2011). The common-mode accelerations, which are produced by the gradiometer in 
Table 6 RMS-of-fit ( $\mathrm{cm}$ ) of SLR tracking observations for the LAGEOS satellites with different gravity field models

\begin{tabular}{lllll}
\hline & EIGEN-5S & GOCE-DIR & GOCE-TIM & GOCE-SPW \\
\hline LAGEOS-1 & 1.98 & 2.05 & 2.05 & 2.00 \\
LAGEOS-2 & 1.80 & 1.87 & 1.88 & 1.82 \\
\hline
\end{tabular}

addition to the gravity gradients, are a good representation of the (remaining) GOCE non-gravitational accelerations and are used in the POD, similar to the approach adopted for CHAMP and GRACE-A/B. Because of the drag-free control system, the non-gravitational accelerations are largely compensated and very small, which leads to very weakly observable scale factors. Therefore, the scale factors for the common-mode accelerations were kept equal to 1 and were not estimated. For GOCE, the estimated parameter set consisted of initial position and velocity (six parameters), three common-mode accelerometer biases ( $X, Y$ and $Z$ directions), and four empirical accelerations to absorb remaining non-static gravitational model errors (e.g. resonances due to tides). It was found that only the GOCE-DIR model leads to a reduction of the 3-dimensional (3D) fit from about 22 to $17 \mathrm{~cm}$ (Table 5). The approach adopted for producing the GOCE-DIR gravity field model thus seems to have (partly) absorbed remaining GOCE orbital resonances not present in the pre-launch model.

Finally, orbits were recomputed with the selected gravity field models for the LAGEOS- 1 and -2 satellites. They orbit at a high altitude of about $6,000 \mathrm{~km}$ and very accurate force models already exist for a long time for these satellites (Noomen et al. 1998). Only a very limited set of parameters is estimated for 8-day arcs, namely initial position and velocity, and 5 empirical accelerations (constant along-track and $1 \mathrm{cpr}$ sine/cosine along-track and cross-track), totalling 11 estimated parameters. It has to be noted that no stationdependent SLR biases or other parameters were estimated. The RMS-of-fit of the SLR observations is about $2 \mathrm{~cm}$ for all models, with slightly better fits for the pre-launch EIGEN-5S model (Table 6). Thus, even the results for LAGEOS corroborate the lower performance of the first release of GOCE gravity field models compared to the pre-launch EIGEN-5S model for precise orbit determination as shown above by ERS-2, CHAMP and GRACE-A/B. This is despite the high altitude of the LAGEOS satellites and the associated lower sensitivity to gravity field perturbations.

It can be concluded that the performance of the GOCEDIR and GOCE-SPW models is comparable to the performance of the EIGEN-5S model for the POD of most selected satellites. In fact, in most cases a slight deterioration can be observed. The GOCE-DIR model performs best for the GOCE POD, which can be explained by the fact that
GOCE data were incorporated in this model by using the same parameter estimation methodology as used for POD (Bruinsma et al. 2010). For the long wavelengths, the GOCE-SPW model is heavily constrained to prior models, which are predominantly based on GRACE data (Migliaccio et al. 2010). The GOCE-TIM model is based solely on GOCE data and was produced from scratch (Pail et al. 2010). Therefore, orbital resonances of other satellites are less well represented by this model explaining the lower performance for the POD of the selected satellites.

\section{GOCE gravity field validation by means of geoid comparisons}

Geoid heights determined by GPS and levelling are often used as one technique to validate global gravity field models (cf. Gruber 2004 for a more general overview of such validation techniques). It is inherently assumed that the quality of ground-based geoid observations is superior compared to those derived from Earth gravity field models. As it is shown later this not always is true and needs to be taken into account when interpreting the validation results. Furthermore, the geoid heights selected for validation have not been used as data source for the global model under evaluation. Therefore, such comparisons are only useful in case that high quality and independent ground-based geoid information is available. Another issue to be taken into account when comparing these quantities is, that any gravity field quantity observed on the Earth's surface contains the full spectral signal power, while a global model is limited by its spectral resolution, i.e. the maximum degree and order of the spherical harmonic series of the model. The signal not represented in the global models is commonly defined as omission error. It has to be estimated by independent means in order to reduce the high-frequency signal from the observed geoid heights. In the following sub-sections the characteristics of the geoid data sets applied for GOCE gravity field validation and the strategy on how to compute the omission error are described. Further, the validation procedure and results for the evaluation of the GOCE gravity field models are presented in detail (cf. also Gruber 2009).

\subsection{Basic relations and GPS levelling data}

Geometric heights of a point on the Earth surface above the reference ellipsoid are observed by GPS positioning. Levelling delivers orthometric heights, i.e. the length of the plumb line from the geoid to the point on the Earth surface, or normal heights, i.e. the vertical distance from the ellipsoid to the telluroid (the surface for which the normal potential equals the gravity potential at the Earth's surface). The differences between the geometric and 
orthometric or normal heights are defined as geoid heights or height anomalies/quasi-geoid heights, respectively. Both quantities are equal in the absence of topography, i.e. over the oceans. For a detailed description of the definition of height systems it is referred to Heiskanen and Moritz (1967) and Hofmann-Wellenhof and Moritz (2006), respectively. In order to compare with GPS-levelling geoid heights/height anomalies, one has to compute the associated quantity from the geopotential models referring to the same normal ellipsoid and the same tide system. This is important in order to avoid systematic differences occurring from different ellipsoid parameters or from inconsistent quantities. The general procedure to compute geoid heights/height anomalies from a gravity potential spherical harmonic series is as follows: (1) Rescaling of geopotential spherical harmonic series to GM (gravity constant times Earth mass) and semi-major axis of the reference ellipsoid used for GPS-levelling geoid heights (normal gravity field has to be consistent with geometric reference ellipsoid); (2) Computation of geocentric spherical coordinates from ellipsoidal coordinates of GPS-levelling points (including ellipsoidal height); (3) Evaluation of spherical harmonic series for the spherical coordinates on the Earth's surface; (4) Computation and addition of the correction term to be applied to height anomalies/quasi-geoid heights to determine geoid heights based on the procedure as described in Rapp (1997). The latter processing step only has to be applied in case geoid heights have to be computed, because geoid heights are available at GPS-levelling stations. The correction term is computed using the Software (hsynth_WGS84.f) and the spherical harmonic expansion of the correction term (Zeta-to-N_to2160_egm2008.gz) provided by the EGM2008 development team (see Pavlis et al. 2008; http://earth-info.nga.mil/GandG/wgs84/gravitymod/ egm2008/egm08_wgs84.html).

From the considerations above, it is important to know what type of height information has been applied in the GPSlevelling data sets, or in other words, are there available geoid heights or height anomalies. In this study, we used GPS-levelling derived geoid heights/height anomalies from various countries or regions. These are Australia, Germany, Europe, Canada, Japan and USA. More details about the data are provided in Table 7. As it can be identified most countries use orthometric heights (geoid heights), while for the European data set use is made of normal heights (height anomalies).

\subsection{Omission error: high frequency signal}

The omission error plays a significant role for validating global models with independently observed terrestrial data. As pointed out above, we want to compare two quantities, which contain either full spectral power (GPS-levelling data), or which are a-priori limited in their spectral content (spherical harmonic series). In order to make them spectrally consistent and finally comparable we have to estimate the omitted signal of the global gravity field model by other means. This is not a trivial task, because one needs to know the global gravity field to infinite spatial resolution at any point in the world. In other words, one can regard the omission error problem as a chicken-and-egg problem, which cannot be solved without assumptions and/or approximations. For the estimation of the omission error we make use of the EGM2008 model (Pavlis et al. 2008), which is complete to degree and order 2,159 with additional spherical harmonic coefficients up to degree 2,190 and order 2,159. As this model is based on a multi year GRACE satellite model and state-of-the-art altimetric, terrestrial and airborne gravity field information with 5 arc min spatial resolution, we can assume that a large part of the gravity field signal beyond the spatial resolution observable by GOCE is represented by this model. Nevertheless, in areas with sparse data coverage like Antarctica, central Asia, Africa and South America this model will not deliver sufficiently well the high-frequency gravity field signal. Therefore, validation of global gravity field models would be very challenging for such areas. As we do not have available GPS-levelling observations in these areas, but only in well observed regions like Europe, North America, Australia and Japan, and because high-quality and high-resolution gravity data from these areas have been used in the computation of EGM2008, one can assume that it represents the gravity field signal there quite well (see Huang and Kotsakis 2008). So the general procedure to estimate the omission error and correct for it is as follows: Compute the geoid height/height anomaly signal from EGM2008 from the degree of truncation to 2,190. Subtract the signal from the GPS-levelling geoid heights/height anomalies and compare them with the model to be validated. We are aware that also EGM2008 does not contain the complete spectral content of the gravity field. Therefore, for the German data set the size and the impact of the remaining signal by means of the residual terrain model (RTM) technique is investigated (Hirt et al. 2010). For this area, RTM based geoid estimates for the remaining signal (above EGM2008 spectral content) have been computed by Hirt and were kindly made available (Hirth 2011). For the investigated area the additional RTM omission error can reach a maximum of about $8 \mathrm{~cm}$ for a few GPS-levelling points in mountainous areas. In general the correction for the 675 German GPS-levelling points used in this study is in the range between 1 and $3 \mathrm{~cm}$. This RTM based omission error was additionally subtracted from the remaining signal in Germany so that a quasi complete correction for the omission error can be assumed. For the other regions used in this study, RTM-based corrections are not yet available, but results shown below suggest that they will improve the estimation of the omission error. 
Table 7 Specifications and data coverage of GPS-levelling data sets

\section{Australia \\ 197points \\ Height system: Orthometric heights \\ Reference: G. Johnston and J. Manning 2003, \\ personal communication}

\section{Germany \\ 675points \\ Height system: Normal heights \\ Reference: Ihde and Sacher 2002}

\section{Europe}

1,233points from EUVN-DA project

(Europ. Vertical Network-Densification A)

Height system: Normal heights

Reference: Kenyeres et al. 2007

Canada

430points

Height system: Orthometric heights

Reference: M. Veronneau 2007, personal communication
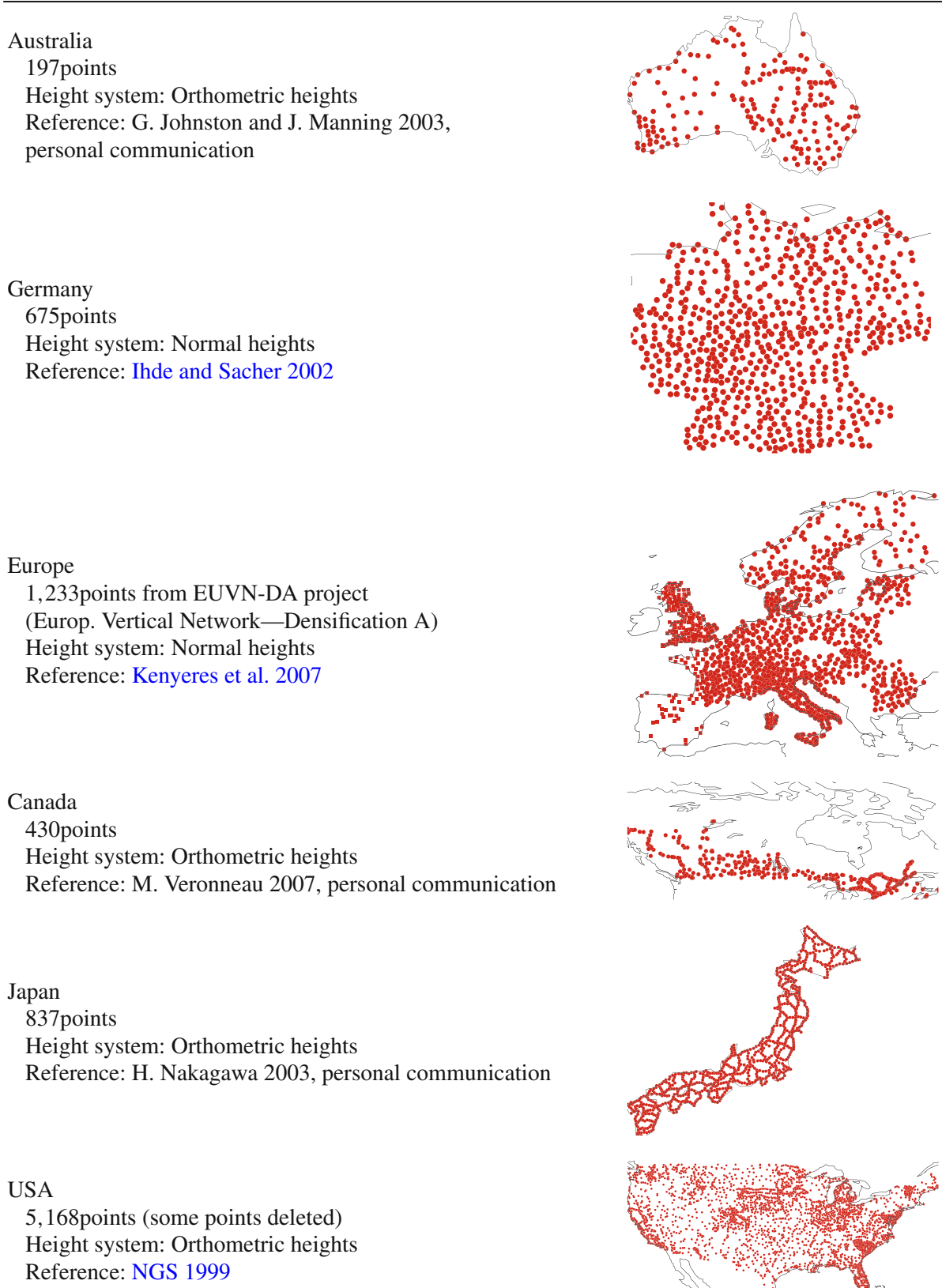

Height system: Orthometric heights

USA

5,168points (some points deleted)

Height system: Orthometric heights

Reference: NGS 1999

Reference: H. Nakagawa 2003, personal communication
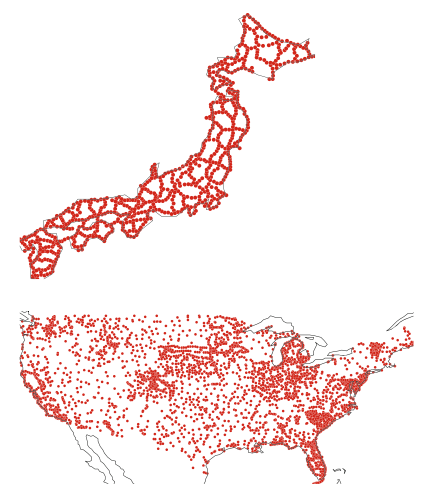

\subsection{Validation procedure}

Using the basic relations and data sets described above, an extensive validation based on GPS-levelling data was performed for the first three HPF GOCE gravity field models as well as some additional (GRACE-based) geopotential models of different kind (see Table 1). Because EGM2008 was used for estimating the omission error it plays a slightly different role in these comparisons (see explanations in the subsequent paragraphs).
The complete procedure to validate global models with independent geoid heights or height anomalies can be described as follows:

1. Geoid heights or height anomalies at GPS-levelling point coordinates up to the degree of truncation $\mathrm{n}_{\max }$ are computed. The tide-free version of the global geopotential models and the WGS84 normal potential was used.,

2. Computation of omission error from EGM2008 and RTM correction (for German data set, c.f. Sect. 3.2). 
3. The omission error is subtracted from the geoid heights and height anomalies at GPS-levelling points.

4. Geoid height and height anomaly differences are computed and maps are produced.

5. For each regional GPS levelling data set a mean value of the differences is computed and subtracted in order to take into account inconsistencies in the height system definitions (or zero potential definition).

6. RMS values of the "un-biased" geoid height/height anomaly differences are computed for each region.

7. Geoid height/height anomaly differences between all possible pairs of GPS-levelling points of a region are computed. Differences between the model and observation (GPS-levelling) differences are computed (subsequently this quantity is named slope difference).

8. Slope differences are more sensitive to mid- to highfrequency variations of the geoid and are sorted with respect to the distance into classes. An RMS per distance class is computed.

The procedure involving steps 1 to 8 was applied for several values of $\mathrm{n}_{\max }$ for the pre-launch and three GOCE gravity field models. Selected results are shown in the next section.

\subsection{Validation results and interpretation}

Geoid height/height anomaly differences are computed for all GPS-levelling points as shown in Table 7. Results are displayed as geographical difference plots as well as in terms of statistics of these differences per regional data set. For the statistics we take into account the mean value of the differences, which commonly is caused by differences in the height datum definitions or other systematic effects. Differences are computed for all data sets for truncation degrees and orders in steps of 10 starting from degree and order 10 to 200 or higher (depending on the resolution of the gravity field model as defined in Table 1). Geoid height/height anomaly slope differences are displayed in terms of RMS of slope differences per distance class. The same degrees of truncation as specified above are applied. As described earlier the EGM2008 model (Pavlis et al. 2008) is used for approximating the omission error. In case of the German data set in addition the RTM based omission error is applied.

\subsubsection{Geoid height/height anomaly differences}

Figure 3 shows the RMS geoid height/height anomaly differences for the selected truncation degrees for all gravity field models and all GPS-levelling data sets applied in this study. The horizontal line for EGM2008 can be regarded as some kind of optimum for a combination of GRACE and surface/altimetry data. In this case the RMS of geoid height/height anomaly differences is constant for all truncation degrees, because the omission error is estimated from the same model and always the complete spherical harmonic series is used. All other models are a mix of the model under investigation (up to truncation degree) and of EGM2008 (for computing the omission error from truncation degree to degree 2,190). For this reason results obtained with EGM2008 shall not be misinterpreted and regarded as some kind of reference baseline. In the subsequent conclusions we concentrate on the other models under test, which all have similar pre-conditions, as far as their consistency is concerned.

From the steepness of the slopes of RMS value curves in Fig. 3, we can identify at what degree a model starts to lose signal. For the GRACE-only models EIGEN-5S and ITG-GRACE2010S this is between degree 120 and 130 and between degree 150 and 170, respectively. The GOCE-only models (GOCE-TIM and to some extent also GOCE-SPW) exhibit more signal content, which can be fully addressed to the 2 months of GOCE gradiometer data used in these models. RMS values for both models strongly increase from about degree 190. In other words, this implies that with 2 months of GOCE gradiometry data more than 7,000 additional spherical harmonic coefficients can be estimated with good signal to noise ratio compared to 7 years of GRACE satellite-tosatellite tracking data $(36,481$ coefficients for degree 190 compared to 29,241 for degree 170 , which corresponds to an increase of parameters of about $25 \%$ ). A slightly different behaviour can be observed for the combined gravity model EIGEN-5C and the GOCE-DIR model, which somehow represents a GOCE add-on to EIGEN-5C. Both models in principle do not lose signal in the test areas under investigation, because terrestrial information entered them. Nevertheless, there are clear indications that GOCE data improve the EIGEN-5C model even up to the full resolution of this model (most RMS values for the GOCE-DIR model are well below EIGEN-5C). For some data sets (e.g. Japan) it seems that the EIGEN-5C model doesn't perform very well for unknown reasons. When adding GOCE data the performance seems to be significantly improved, which is a clear indicator, that in a specific spectral range GOCE can replace terrestrial data in case they exhibit larger uncertainties. This also is supported by slightly improved RMS-values compared to the EGM2008 performance for some lower truncation degrees in some test areas. So even if we do not expect sensitivity of GOCE up to degree 240, higher quality data and improved data consistency from GOCE can increase the quality of combined satellite and terrestrial high-resolution models.

Looking at the range of the $y$-axes in Fig. 3, we can identify that it varies for each GPS-levelling data set. From this we can get some information about the overall quality and consistency of the regional GPS-levelling data sets, specifically when looking to the low truncation degrees, where we have very good performance from GRACE and where all models 

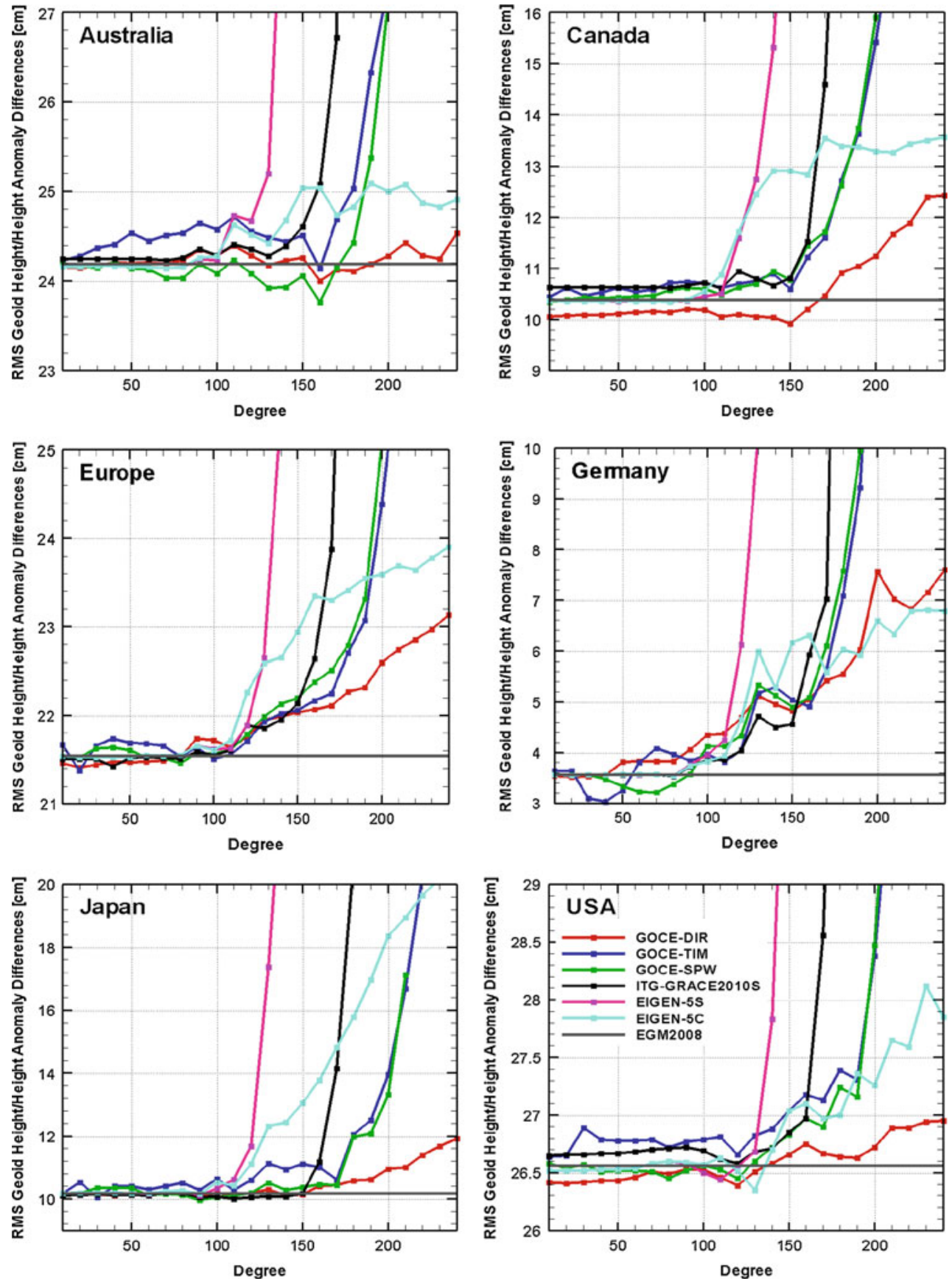

Fig. 3 RMS geoid height differences (height anomaly differences in case of European and German data set) after subtraction of mean value in $(\mathrm{cm})$ for the selected truncation degrees for GOCE and other global

gravity field models. Colour code identical in all sub-figures: red GOCEDIR, blue GOCE-TIM, green GOCE-SPW, black ITG-GRACE2010S, magenta EIGEN-5S, cyan EIGEN-5C, grey EGM2008)

are very similar in performance. Specifically the horizontal line for the EGM2008 comparisons somehow represents the minimum error level, which can be reached with a regional GPS-levelling data set. As it can be identified there is some relation between the test area size and the minimum error level. Because of homogeneity and vertical datum issues, a continent-wide data set by nature shows a higher error level

than one for a smaller country. From the results obtained the best data set available for this study is the German data set with a minimum value for the RMS differences of about $3.5 \mathrm{~cm}$. Good performance is reached with the data sets from Japan (10 cm minimum RMS) and Canada $(10.5 \mathrm{~cm})$, while the Australian, European and U.S. data sets show quite large difference RMS values $(24,21.5$ and $26.5 \mathrm{~cm}$, respectively). 
Fig. 4 Height anomaly differences of GOCE gravity field models for Germany for two different degrees of truncation. Top row GOCE-DIR degree 150 (left), GOCE-DIR degree 170 (right). Middle row GOCE-TIM degree 150 (left), GOCE-TIM degree 170 (right). Bottom row GOCE-SPW degree 150 (left), GOCE-SPW degree 170 (right)
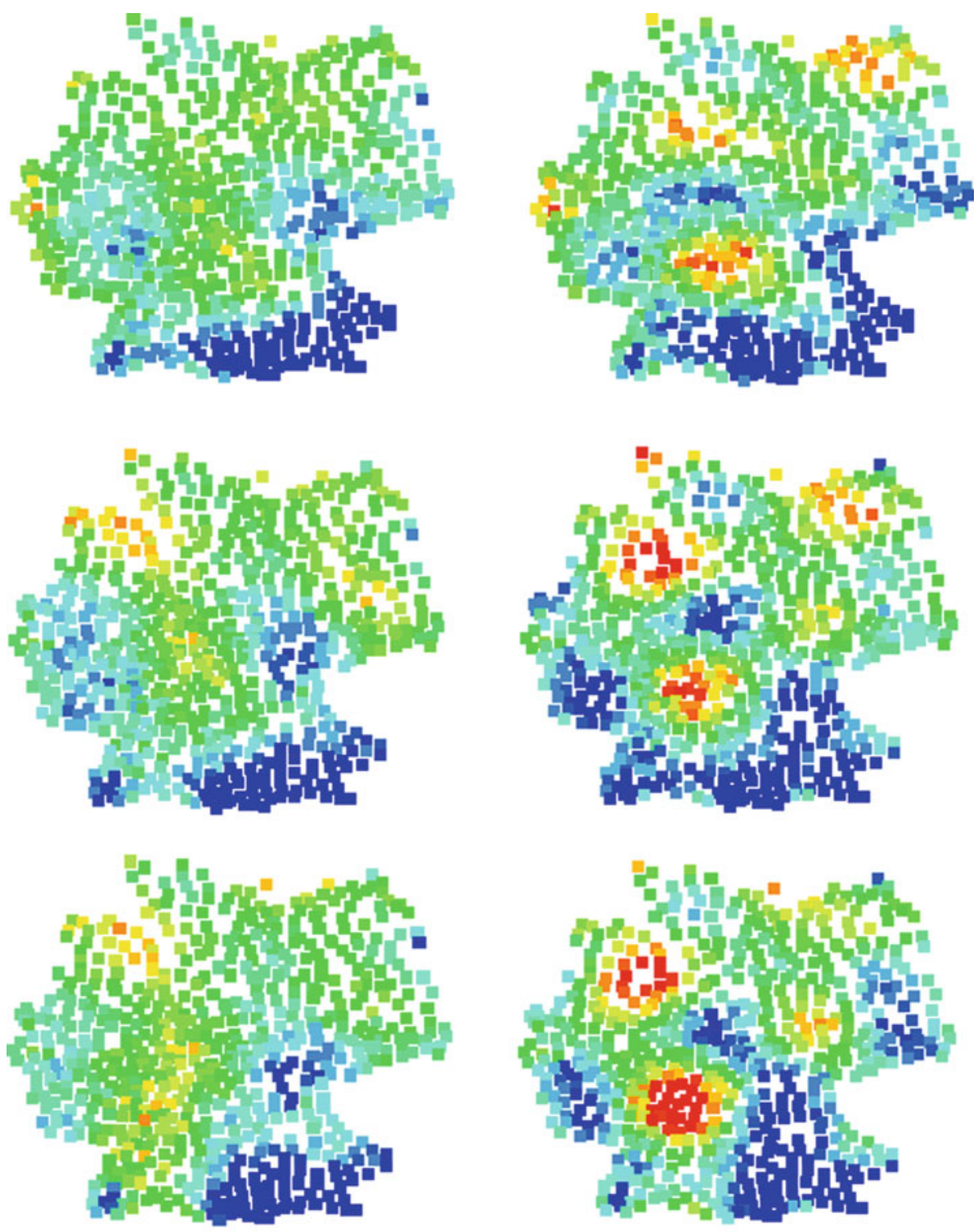

From this we can conclude that for further investigations, the German, Japanese and Canadian data should be applied. Figure 4 shows the height anomaly differences for Germany with truncation degrees of 150 and 170 for the three GOCE models, while Fig. 5 shows the same for two GRACEbased models (ITG-GRACE2010S and EIGEN-5C) and EGM2008. For all differences, the omission error is taken into account and the mean value is $-33 \mathrm{~cm}$.

From Figs. 4 and 5 it can be concluded that for a truncation degree 150 the pure GOCE as well as the EIGEN5C-based GOCE solutions (Fig. 4 left column) are well in agreement with the pure GRACE solution in Germany (Fig. 5 top left). As the pure GRACE solution is significant at least up to degree 150 (c.f. Fig. 3 middle right) this indicates that

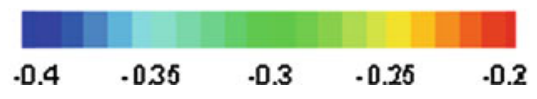

the GOCE solutions have good performance in this spectral band (up to 150) and that the remaining systematic differences (e.g. slope from North-West to South-East) may indicate systematic uncertainties in the GPS-levelling data set. It is remarkable that the height anomaly differences for the EIGEN-5C model look noisier with some systematic pattern, while by adding GOCE data to this model this feature could be reduced significantly (GOCE-DIR solution in Fig. 4 top left). The situation changes when truncating the global gravity field models at degree 170. In all three GOCE models, some bumps can be identified, which are more pronounced for the pure GOCE solutions GOCE-TIM and GOCE-SPW (c.f. Fig. 4 middle right and bottom right) compared to GOCEDIR, which is based on EIGEN-5C. Obviously the observed 
Fig. 5 Height anomaly differences of GRACE based gravity field models for Germany for two different degrees of truncation. Top row ITG-GRACE2010S degree 150 (left), ITG-GRACE2010S degree 170 (right). Middle row EIGEN-5C degree 150 (left), EIGEN-5C degree 170 (right). Bottom row EGM2008 (left). Note Because EGM2008 was applied for estimating the omission error height anomaly differences for this model are identical for all truncation degrees
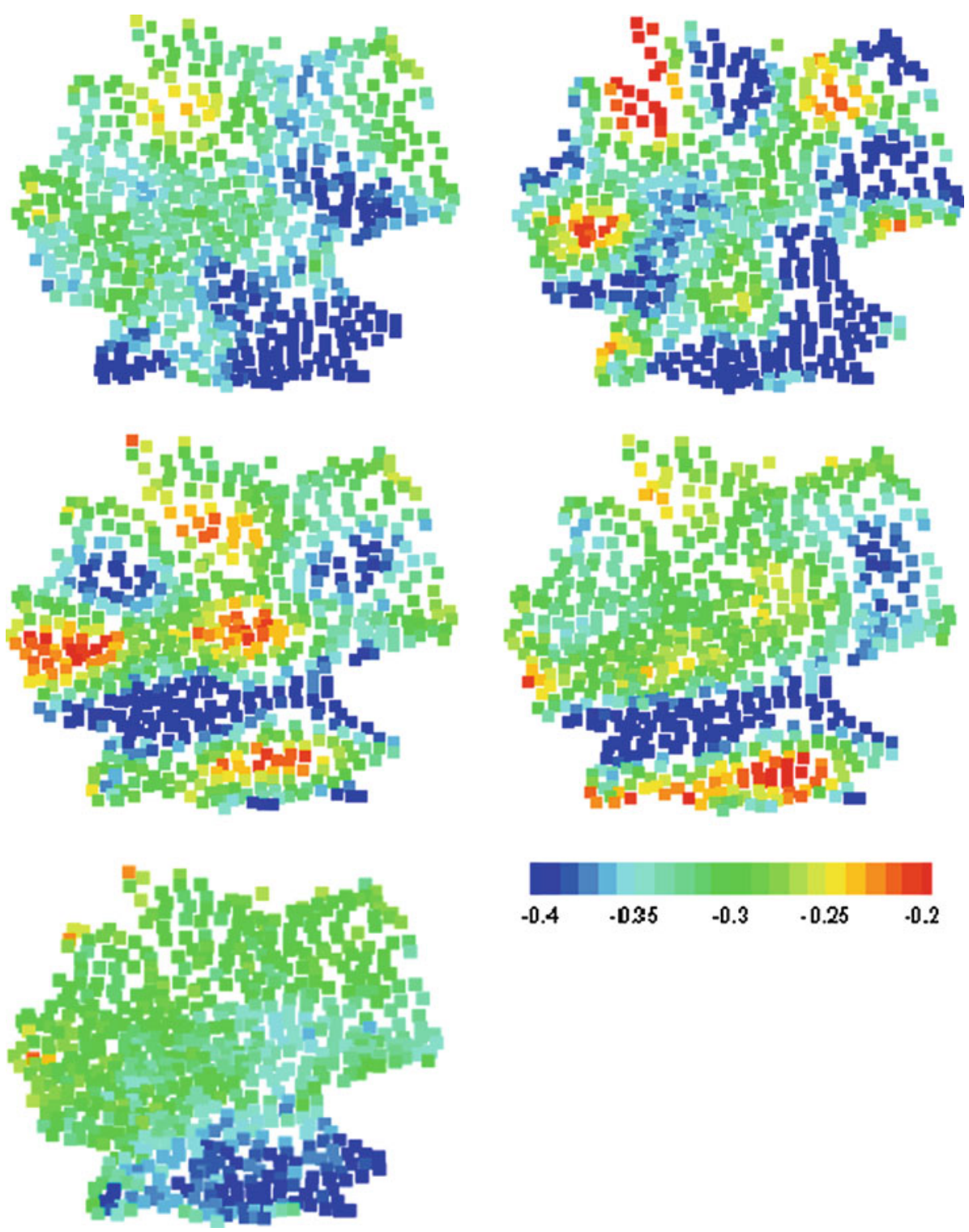

GOCE gradients do not fully reflect the gravity field signal in this spectral range so that some artificial features show up in the global solutions. From this investigation it can be concluded that in an area with a low varying geoid signal (like in Germany) the 2 months of GOCE data provide some additional value, but that the 7 years GRACE solution also performs very well. With more GOCE data to be used in future, it is expected that the performance of the GOCE models will increase for higher degrees as well.

\subsubsection{Geoid height/height anomaly slope differences}

Geoid height/height anomaly slope differences are more sensitive to mid- to high-frequency variations of the geoid.
Therefore, we take a closer look at the results obtained for the German and the Japanese data sets. For the other data sets similar, but less pronounced results as described in the following were obtained. Figure 6 shows the RMS per distance class for a number of truncation degrees (140, 160, 180 and 200) for both regions. EGM2008 again was included as reference model. First of all it can be identified that for degree 140, apart from EGM2008, the pure GRACE model ITGGRACE2010S performs best, while at degree 160 it starts to lose signal (therefore this model is not anymore shown for degrees 180 and 200). EGM2008 shows very good performance for the German data set and also outperforms all other models in Japan for higher truncation degrees. What concerns this model, the same comment about consistency and mixing coefficients holds as for the results presented in Fig. 3. Nevertheless, results indicate that the combination 
Fig. 6 RMS of geoid slope differences per distance class for Germany (left column) and Japan (right column) for various degrees of truncation. Colour code identical in all sub-figures: red GOCE-DIR, blue

GOCE-TIM, green

GOCE-SPW, black

ITG-GRACE2010S, cyan

EIGEN-5C, grey EGM2008).

Note the different $y$-scale for the geoid slope differences in Germany
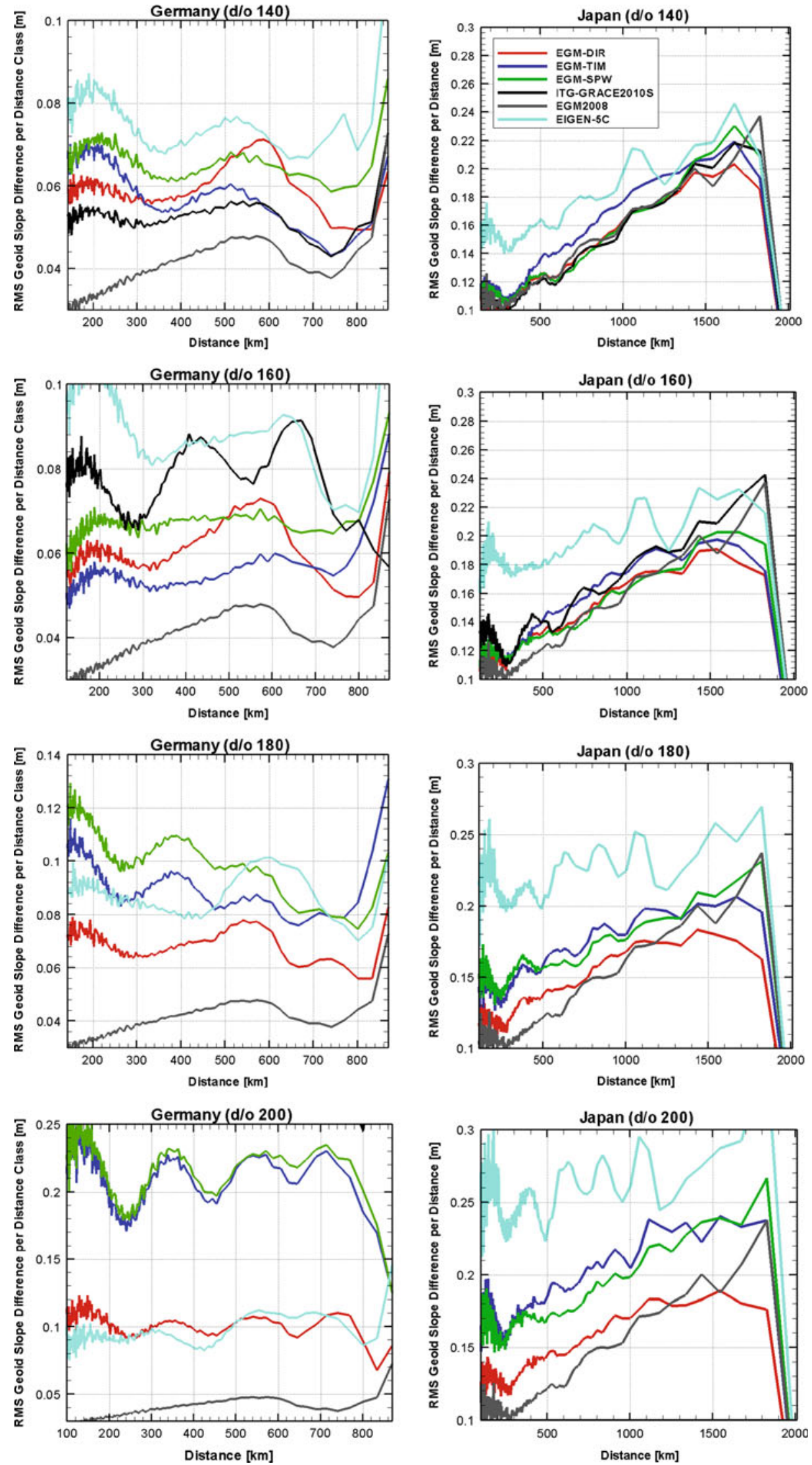
of GRACE information with terrestrial data was performed very consistently during the EGM2008 computation, while the opposite is visible for the EIGEN-5C model. The RMS slope differences for the EIGEN-5C model, for all truncation degrees, remain at a similar level for Germany (note the different scales on the $y$-axes for the left column in Fig. 6), while they are increasing with the degree of truncation for the Japanese data set. The RMS slope differences for this model show some strange wave features, which probably indicate some problem in the data combination approach or in the terrestrial data used for EIGEN-5C. Furthermore, some other models investigated show similar features, which in case of satellite-only models (ITG-GRACE2010S and GOCETIM) most likely are caused by not represented signals in these models. Looking to the GOCE models we can conclude that at degree 140 they slightly perform worse than the pure 7 years' GRACE model, while for higher degrees (from 150 to 170 ) they outperform GRACE (specifically visible for truncation degree 160-2nd row in Fig. 6). At truncation degree 180 and higher, we can identify that the pure GOCE models do not contain the full signal anymore. Nevertheless, for the Japanese data set they show a remarkably good performance. Again, the GOCE adjusted EIGEN-5C model (GOCE-DIR) displays a significantly improved performance at the high degree terms when compared to the base model. For Japan, even at truncation degree 200 it performs better, while for Germany it stays on a similar level as EIGEN-5C. Hence, a similar conclusion as above can be drawn: GOCE data strongly support the combination of satellite and terrestrial data by introducing new consistent medium frequency gravity field information. This will help significantly to derive improved combined high-resolution global gravity field models.

\section{Summary and conclusions}

Three GOCE-based global gravity field models computed with different processing strategies have been analyzed in terms of orbit and geoid residuals in order to determine their quality and accuracy. While orbital perturbations are mostly sensitive to the long wavelengths, geoid comparisons reflect the medium to short wavelength quality (degree 50-200). Sections 2 and 3 describe in detail the techniques applied and show the results obtained. From them the following can be concluded.

1. Precise orbit determination results indicate that great care has to be taken when combining GOCE and GRACE data for determining the long wavelength part of the Earth's gravity field. Compared to pre-launch models, all first three released GOCE HPF gravity field models show a degraded performance in precise orbit determination for the selected satellites, the only exception being the GOCE-DIR model for computing the GOCE orbit itself. For GOCE, the long wavelength information is derived from the GPS observations or, more specifically, the GPS-derived orbit perturbations. It can be concluded that this information is not yet optimally used in the combined models (GOCE-DIR and GOCE-SPW). Depending on the processing strategy and the amount of a-priori information included in the GOCE solutions, tracking residuals and orbit fits as well as single- and dualmission altimeter crossovers increase. It turns out that the GOCE-DIR and the GOCE-SPW models, which both are using a-priori information from GRACE, exhibit slightly increased residuals, while the GOCE-TIM model, which is computed from scratch from 2 months of GOCE data only shows significantly larger residuals. These results are confirmed for all chosen satellites for which tracking residuals, altimeter crossover residuals or orbit fits are computed (ERS-2, CHAMP, GRACE-A/B and LAGEOS-1/2). It is important to note that improved GOCE orbit fits are obtained with the GOCE-DIR model. Concerning orbit perturbations, the approach adopted for producing this model is the only one that aims at optimally fitting GPS-derived GOCE orbits, explaining this improved performance. This indicates that there is some potential in the GOCE data to improve some harmonic orders of the spherical harmonic series, which are related to the resonant frequencies of the GOCE orbit. Also, there are indications that more resonant coefficients need to be estimated (i.e. to higher degree and order) to avoid polluting other gravity field coefficients by these very resonances. In conclusion, a proper weighting of the GOCE information and a proper parameterization could help to improve gravity fields for the long wavelengths as well. A deeper investigation of this is needed when more GOCE gravity data become available.

2. GOCE data provide significant new information for the medium to higher spatial scales of the Earth's gravity field. This is supported by the results obtained from the comparison of geoid heights computed from these models with independent information from GPS-levelling points. Using different truncation degrees of the global models we can identify up to which degree the models provide significant results, or in other words when do they start to lose signal due to the attenuation of the gravity signal with satellite height. When analyzing the results obtained with the high-quality GPS-levelling data in Germany it can be concluded that the geoid accuracy is at the level of $7 \mathrm{~cm}$ at degree and order 180 (corresponding to a resolution of $111 \mathrm{~km}$ in the spatial domain). Taking into account the error level of the GPS-levelling data of about 3-4 cm (as it can be derived from the 
EGM2008 baseline error level in Fig. 3 mid right) it can be assumed that with the 2 months of GOCE data a global geoid accuracy of about 5-6 cm with this spatial resolution can be reached. This is not yet the mission goal of $1-2 \mathrm{~cm}$ geoid accuracy with $100 \mathrm{~km}$ resolution, but with the availability of more data it can be assumed that the goal is reachable and that GOCE data provide a highly valuable data set. For the GOCE-DIR solution even a better accuracy than for the other GOCE based solutions can be reached. The reason for this is that this model is based on a combined GRACE/terrestrial a-priori model (EIGEN-5C). Therefore, for the higher frequencies, terrestrial data come into play and provide additional highfrequency signal. Nevertheless, it is remarkable that by adding GOCE data to the combined a-priori model a significant improvement for the geoid comparisons can be observed, specifically in areas where the geoid knowledge is not as good as for example in Germany. This indicates the high value of the GOCE data to determine the global geoid consistently with high accuracy.

In summary, it can be stated that with the global gravity field models based on only 2 months of GOCE data a significant step towards the GOCE mission goal was achieved. It can be assumed that the expectations of the mission can be fulfilled when a longer time series of high-quality GOCE data will become available and incorporated into global gravity field models as well as from lessons learned from the processing of the initial data set. Results of orbit and tracking observation fits and geoid tests clearly indicate that a combination of all satellite data will lead to the best performance of the gravity field solutions. Even if not a primary mission goal, GOCE data can contribute to a consistent determination of the gravity potential spherical harmonic coefficients in the long wavelength spectrum as well. In the medium to low wavelength spectrum (in the range of degree and order 50 to 200 in terms of spherical harmonics), GOCE will be a unique consistent data source and will repair artefacts in current combined gravity field models due to low quality terrestrial information.

Acknowledgments We are very thankful to three anonymous reviewers who helped to improve the manuscript significantly. GPS levelling data have been provided for validation purposes by AUSLIG, BKG, the Japanese Geographical Survey Institute, NGS and Natural Resources of Canada. The provision of these data is highly appreciated by the authors. We are thankful to $\mathrm{Ch}$. Hirt, who provided RTM estimates for the omission error for the German GPS-levelling data set. The NASA Goddard Space Flight Center is acknowledged for kindly providing the GEODYN software.

\section{References}

Altamimi Z, Colillieux X, Legrand J et al (2007) ITRF2005: a new release of the International Terrestrial Reference Frame based on time series of station positions and Earth orientation parameters. J Geophys Res 112(B9):B09401

Bock H, Jaggi A, Meyer U, Visser P, van den IJssel J, Van Helleputte T, Heinze M, Hugentobler U (2011) GPS derived orbits for the GOCE satellite. J Geod (this issue)

Bruinsma SL, Marty JC, Balmino G, Biancale R, Foerste C, Abrikosov O, Neumayer H (2010) GOCE gravity field recovery by means of the direct numerical method. In: Proceedings of the ESA living planet symposium, 28 June-2 July 2010, Bergen, Norway. See also: http://earth.esa.int/GOCE

ESA (2010) http://www.esa.int/SPECIALS/GOCE/SEMY0FOZVAG _0.html

Förste Ch, Flechtner F, Schmidt R, Stubenvoll R, Rothacher M, Kusche J, Neumayer H, Biancale R, Lemoine J-M, Barthelmes F, Bruinsma S, Koenig R, Meyer Ul (2008) EIGEN-GL05C: a new global combined high-resolution GRACE-based gravity field model of the GFZ-GRGS cooperation. Geophysical Research Abstracts, vol 10. EGU2008-A-03426，2008 SRef-ID: 1607-7962/gra/EGU2008A-03426, 2008

Gruber Th (2004) Validation concepts for gravity field models from satellite missions. In: Lacoste $\mathrm{H}$ (ed) Proceedings of the 2nd international GOCE user workshop "GOCE, The Geoid and Oceanography”, ESA SP-569, ESA. ISBN (Print):92-9092-880- 8, ISSN:1609-042X

Gruber Th (2009) Evaluation of the EGM2008 gravity field by means of GPS-levelling and sea surface topography solutions. Newton's Bull Bur Gravimétrique Int (BGI)/Int Geoid Service (IGeS) 4:3-17

Gruber Th, Rummel R, Abrikosov O, van Hees R (2010) GOCE level 2 product data handbook, issue 4.2. ESA

Heiskanen WA, Moritz H (1967) Physical geodesy. W.H. Freeman \& Co Ltd, New York

Hirt C, Featherstone W, Marti U (2010) Combining EGM2008 and SRTM/DTM2006.0 residual terrain model data to improve quasigeoid computations in mountainous areas devoid of gravity data. J Geod 84(9):557-567. doi:10.1007/s00190-010-0395-1

Hirth C (2011) Assessment of EGM2008 over Germany using accurate quasigeoid heights from vertical deflections, GCG05 and GPS levelling. Zeitschrift für Geodäsie, Geoinformation und Landmanagement (zfv) (accepted)

Hofmann-Wellenhof B, Moritz H (2006) Physical geodesy, 2nd edn. Springer, Wien

Huang J, Kotsakis C (eds) (2008) External quality evaluation reports of EGM08. Newton Bulletin no. 4. International Association of Geodesy and International Geoid Service. ISSN:1810-8555

IERS International Earth Rotation Service (2008) http://www.iers.org

Ihde J, Sacher M (2002) EUREF publication 11/I, vol 25. Mittelungen des Bundesamtes für Kartographie und Geodäsie, Frankfurt/Main

Kenyeres A, Sacher M, Ihde J, Denker H, Marti U (2007) EUVN_DA: establishment of a European continental GPS/levelling network. In: Proceedings of the 1st international symposium of the international gravity field service, Istanbul 2006. Harita Dergisi, special issue, no. 18. ISSN:1300-5790

Knudsen P, Bingham R, Andersen O (2011) Enhanced mean dynamic topography and ocean circulation estimation using GOCE preliminary models. J Geod (this issue)

Mayer-Gürr T, Kurtenbach E, Eicker A (2010) http://www.igg. uni-bonn.de/apmg/index.php?id=itg-grace 2010

Migliaccio F, Reguzzoni M, Sanso F, Tscherning CC, Veicherts M (2010) GOCE data analysis: the space-wise approach and the first space-wise gravity field model. In: Proceedings of the ESA living planet symposium, 28 June-2 July 2010, Bergen, Norway. ESA SP-686. See also: http://earth.esa.int/GOCE

NGS (1999) http://www.ngs.noaa.gov/GEOID/GPSonBM99/gpsbm 99.html

Noomen R, Ambrosius BAC, Leenman H, Wakker KF (1998) Precise orbit computations of LAGEOS. In: AIAA-88-4213-CP, 
AIAA/AAS astrodynamics conference, Minneapolis, Minnesota, August 15-17, 1998

Pail R, Goiginger H, Mayrhofer R, Schuh WD, Brockmann JM, Krasbutter I, Höck E, Fecher T (2010) GOCE gravity field model derived from orbit and gradiometry data applying the time-wise method. In: Proceedings of the ESA living planet symposium, 28 June-2 July 2010, Bergen, Norway. ESA SP-686. See also: http:// earth.esa.int/GOCE

Pail R, Bruinsma S, Migliaccio F, Förste Ch, Goiginger H, Schuh W-D, Höck E, Reguzzoni M, Brockmann JM, Abrikosov O, Veicherts M, Fecher T, Mayrhofer R, Krasbutter I, Sansò F, Tscherning CC (2011) First GOCE gravity field models derived by three different approaches. J Geod (this issue)

Pavlis DE, Poulouse S, McCarthy JJ (2006) GEODYN operations manual. Contractor report. SGT Inc., Greenbelt

Pavlis NK, Holmes SA, Kenyon SC, Factor JK (2008) An Earth gravitational model to degree 2160: EGM2008. Presented at the 2008 general assembly of the European Geosciences Union, Vienna, Austria, April 13-18, 2008

Rapp RH (1997) Use of potential coefficient models for geoid undulation determinations using a spherical harmonic representation of the height anomaly/geoid undulation difference. J Geod 71: 282-289

Rummel R, Gruber T, Koop R (2004) High level processing facility for GOCE: products and processing strategy. In: Lacoste H (ed)
Proceedings of the 2nd international GOCE user workshop "GOCE, the geoid and oceanography", ESA SP-569

Scharroo R, Visser PNAM (1998) Precise orbit determination and gravity field improvement for the ERS satellites. J Geophys Res 103(C4):8113-8127

van den IJssel J, Visser PNAM (2003) Champ precise orbit determination using GPS data. Adv Space Res 31(8):1889-1895

Van Helleputte T, Visser P (2007) GPS based reduced dynamic orbit determination using accelerometer data. In: Stengle T (ed) 20th International symposium on space flight dynamics, September 24-28, 2007. NASA/CP-2007-214158, Annapolis, MD, USA, pp $1-7$

Visser PNAM (2005) ERS-2 orbit computations with CHAMP- and GRACE-based gravity field models. Adv Space Res 36(3): 454-459

Visser PNAM, Scharroo R, Ambrosius BAC (2002) Application of ERS-2 PRARE data for orbit determination and gravity field and station coordinate estimation. Adv Space Res 30(2):249-254

Visser PNAM, van den IJssel J, Van Helleputte T, Bock H, Jaeggi A, Beutler G, Hugentobler U, Svehla D, Heinze M (2009) Orbit determination for the GOCE satellite. Adv Space Res 43(5): 760-768. doi:10.1016/j.asr.2008.09.016 